\title{
Galactic disc formation from cold fractal gas
}

\author{
B. Semelin \\ Dep of Physics, Waseda Univ, Oh-kubo, Shinjuku-ku, Tokyo 169-8555, \\ Japan \\ F. Combes \\ DEMIRM, Observatoire de Paris, 61 av de l'Observatoire, 75014, Paris, \\ France
}

\begin{abstract}
.
It is likely that cold molecular clouds formed early, even before galaxies. In this work, we try to assess the consequences of using cold fractal gas for disc formation instead of hot gas, with a focus on angular momentum loss through dynamical friction. Using numerical simulations, we show that the dynamical friction from the dark matter halo on the cold gas fractal structures is not affected by the morphology of the structures, but is affected by their dynamical nature: their deformations and fluctuations. We argue that for typical values of the physical parameters during disc formation, the friction at small scale is reduced .
\end{abstract}

\section{Introduction}

There is evidence that cold fractal molecular clouds form at high redshift, before galaxies. In this work, we examine how the physics of disc formation is modified when we consider cold gas as the basic material. Indeed, until now, numerical simulations have assumed that the infalling gas is hot and rather homogeneous, cooling being artificially stopped at $10^{4} \mathrm{~K}$.

One the most sensitive issues in numerical disc formation is the angular momentum problem. Several processes have been tested to alleviate the problem (e.g. Navarro \& Steinmetz 1997). Our approach is to examine whether cold fractal gas reacts differently to the dynamical friction from the halo.

Formation of fractal clumps at high redshift requires two conditions: efficient early cooling, leading to isothermal collapse of initial density fluctuations; and inefficient star formation, to avoid over production of population III stars ( Combes \& Pfenniger 1998).

\section{Results}

For the simulations we use a parallel treecode, and consider up to $10^{6}$ particles. In addition to gravity, dissipation in the baryonic component is obtained through inelastic collisions. Periodic boundary conditions are used, since we set the 


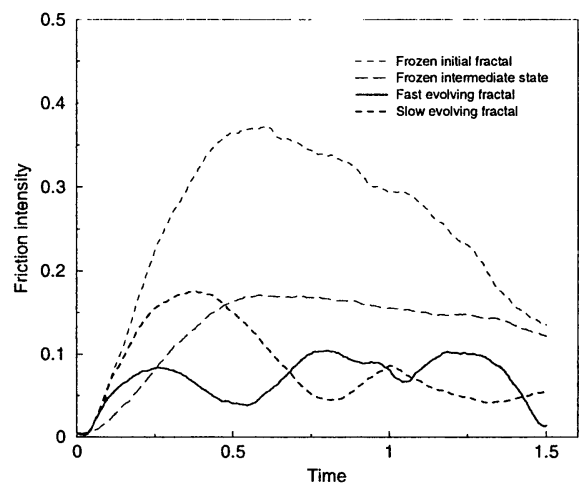

Fig 1: Intensity of the dynamical friction on dynamic cold fractal gas as a fucntion of time. The four plots are for 2 frozen states of the gas, one slowly evolving, and one rapidly evolving state. The evolving states induce a weaker friction.

simulation box to a small part of the forming disc. We consider two components: collisionless dark matter particles and dissipative gas.

A first simulation shows that, for typical disc formation conditions, the dynamical friction is not strong enough to drive the GMC structure, which is regulated by the self-gravity of the clouds.

To study the friction on time scale longer than the clouds dynamical time, we must stabilize the fractal structure. This is a complex issue (Semelin and Combes (2000)). Here, we choose to freeze the structure. We isolate an artificial, frozen, GMC-like structure within a drifting background, and modify the fractal parameters to study their influence on the intensity of the friction. We find that the presence of substructures in the GMC has little influence on the total intensity of the friction (less than $1 \%$ variation). The branching ratio and fractal dimension do not have any systematic effect either. Consequently the friction is not affected by the static morphology of the fractal.

We now study the influence of the fluctuating nature of the structure of GMCs. We impose an artificial dynamics to a fractal structure: a linear transformation between two fractal states obtained from simulations. We add a drifting dark matter background obeying the induced dynamics. Temporary gravitational wakes form and fade along with clumps. Measuring the total friction exerted on the gas we show that it decreases when the fluctuation time of the fractal structures decreases: the wakes do not have time to form. Considering relevant values for the fluctuation of GMCs (crossing time: $10 \mathrm{Myr}$ ), and typical build-up time of wakes, ( $5 \mathrm{Myr}$ for 500 pc maximum impact parameter and 100 $\mathrm{km} . \mathrm{s}^{-1}$ drifting speed), we show that this weakening of the friction is relevant. However this applies on scales below usual resolution of galaxy formation simulations. Consequently it mainly insures that the angular momentum problem will not worsen with higher resolution.

Acknowledgments. B. Semelin work was supported by a JSPS fellowship.

\section{References}

Combes, F. \& Pfenniger, D. 1998, Mem Soc Astron Italiana, 69, 413

Navarro, J. F., \& Steinmetz, M. 1997, ApJ, 478, 13

Semelin, B., \& Combes, F. 2000, A\&A, 360,1096 